Pure and Applied Mathematics Quarterly

Volume 4, Number 4

(Special Issue: In honor of

Jean-Pierre Serre, Part 1 of 2)

$1107-1132,2008$

\title{
On Local Constants Associated to Arithmetical Automorphic Functions
}

\section{P. Bayer and A. Travesa}

Dedicated to Jean-Pierre Serre on the occasion of his 80th birthday

\begin{abstract}
This article deals with the diophantine nature of the local constants associated to automorphic functions with respect to Fuchsian groups obtained from quaternion algebras. We focus on the canonical model of those curves arising from the quaternion $\mathbb{Q}$-algebra of discriminant $D=6$.
\end{abstract}

\section{INTRODUCTION}

Some of the most useful Shimura varieties arise as moduli spaces of abelian schemes with extra structures, in the same way as modular curves are moduli spaces of elliptic curves. In the 1960s, a convenient generalization of the complex multiplication theory for the modular case led Shimura to his theory of canonical models (cf. [21], and [24] for a recent account of the theory).

One of the first applications of Shimura curves was provided in [13]. By using fine properties of the Néron models of the jacobians of modular curves and of Shimura curves associated to quaternion algebras, Ribet was able to prove a very particular case of a deep conjecture of Serre relative to the modularity of (mod p) Galois representations of dimension 2, stated in [19]. As is well known, this was the starting point of Wiles' proof of Fermat's Last Theorem (see for instance [20]). At present, very intensive work has been carried out towards a general proof of Serre's conjecture.

Received July 18, 2006.

1991 Mathematics Subject Classification. 11F03, 11G18, 14G35, 30F35.

Partially supported by MEC, MTM2006-04895. 
In spite of their increasing interest, Shimura varieties have been much less studied from an effective point of view, even in dimension one. The reasons appear to be the lack of cusps and, consequently, the lack of Fourier expansions for the automorphic functions involved. In contrast, modular functions possess $q$-expansions at infinity, being $q(z)=e^{2 \pi i z}$, which were already known by the classics.

In our previous articles [3] and [6], we considered the canonical models of Shimura curves associated to the indefinite quaternion $\mathbb{Q}$-algebra of discriminant $D=6$. We expanded their functions at elliptic points and at some distinguished CM-points, called special (SCM for short).

This article is concerned with the characterization of the $\overline{\mathbb{Q}}$-rational functions of the canonical models through their expansions at CM-points. We will restrict our attention to the case of the quaternion $\mathbb{Q}$-algebra of discriminant $D=6$.

The article is organized as follows:

In sections 1 and 2 we summarize the contents of [6] to make our exposition self-contained. The functions defining the canonical models are introduced as solutions of non-linear differential equations of the third order which are set up from fundamental domains.

In section 3 we derive an interesting formula which expresses a particular quaternionic automorphic function through quotients of Siegel automorphic forms. In some sense, the formula is analogous to the one expressing the elliptic $j$ function in terms of Eisenstein series (cf. [18]).

In sections 4 and 5 we introduce some local parameters and obtain explicit expansions at the elliptic points and SCM-points for the functions in section 2 . The local parameters adapted to these functions turn out to be of the form

$$
q_{P}(z)=\left(k_{P} \frac{z-P}{z-\bar{P}}\right)^{e_{P}},
$$

$e_{P}$ being the order of the isotropy group at $P$ and $k_{P}$ a constant of the same transcendence class as certain products of values of Euler's gamma function at rational arguments.

In section 6 it is proved that the constants $k_{P}$ agree, up to algebraic elements, with some specific constants $\pi_{d}$ which only depend on the discriminant $d$ of the field of complex multiplication. The identification of the constants at the elliptic points and at the SCM-points involves the use of the Chowla-Selberg formula [17]. The transcendence of the $\pi_{d^{-}}$s, for every $d$, is established by means of a theorem due to Chudnovsky [8].

The results in section 6 are generalized to every CM-point in section 7. Theorem 7.1 illustrates a theorem due to Shimura ([23], theorem 7.1) and shows that 
the transcendental part of $k_{P}$ at a CM-point $P$ agrees with $\pi_{d}$. Finally, in theorem 7.3 we stress the arithmetical meaning of the parameters $\pi_{d}$ that is, that they allow the characterization of the canonical models through series expansions.

\section{Contents}

Introduction

1. Preliminaries on the curve $X_{6}$

2. Canonical models

3. Uniformizing functions and theta constants

4. Local parameters

5. Local expansions

6. Transcendence classes of adapted local constants at elliptic points and at SCM-points

7. Arithmetical properties of the canonical models

References

\section{Preliminaries on the Curve $X_{6}$}

Let $\mathrm{H}_{6}$ be the indefinite quaternion $\mathbb{Q}$-algebra of discriminant 6 , endowed with a basis $\{1, \mathrm{I}, \mathrm{J}, \mathrm{IJ}\}$ such that $\mathrm{I}^{2}=3, \mathrm{~J}^{2}=-1$, IJ $=-\mathrm{JI}$. From the main antiinvolution of the algebra

$$
\alpha=x+y \mathrm{I}+z \mathrm{~J}+t \mathrm{IJ} \mapsto \bar{\alpha}=x-y \mathrm{I}-z \mathrm{~J}-t \mathrm{IJ}, \quad x, y, z, t \in \mathbb{Q},
$$

the reduced trace and reduced norm of an element $\alpha \in \mathrm{H}_{6}$ are defined by

$$
\operatorname{Tr}(\alpha)=\alpha+\bar{\alpha}, \quad \mathrm{N}(\alpha)=\alpha \bar{\alpha} .
$$

We consider the order $\mathcal{O}_{6}:=\mathbb{Z}[1, \mathrm{I}, \mathrm{J},(1+\mathrm{I}+\mathrm{J}+\mathrm{IJ}) / 2]$ as a representative of the conjugacy class of all maximal orders of $\mathrm{H}_{6}$.

Throughout the paper we fix the embedding of algebras

$$
\begin{aligned}
& \Phi: \quad \mathrm{H}_{6} \quad \longrightarrow \quad \mathbf{M}(2, \mathbb{R}) \\
& x+y \mathrm{I}+z \mathrm{~J}+t \mathrm{IJ} \mapsto\left[\begin{array}{cc}
x+y \sqrt{3} & z+t \sqrt{3} \\
-(z-t \sqrt{3}) & x-y \sqrt{3}
\end{array}\right] .
\end{aligned}
$$

The group of units of $\mathcal{O}_{6}$ of reduced norm equal 1 can be identified with its image $\Gamma_{6} \subseteq \mathbf{S L}(2, \mathbb{R})$ under $\Phi$. The group $\Gamma_{6}$ and its projection $\bar{\Gamma}_{6}:=\Gamma_{6} /\{ \pm 1\}$ 
in $\mathbf{P S L}(2, \mathbb{R})$ are Fuchsian groups of the first kind without parabolic transformations. The action of $\bar{\Gamma}_{6}$ on the complex upper half-plane $\mathcal{H}$ by homographies yields a projective non-singular curve of genus zero.

Let us consider an imaginary quadratic field $K$ and suppose that there is an embedding $\varphi: K \hookrightarrow \mathrm{H}_{6}$. The embedding $\varphi$ determines a point $P \in \mathcal{H}$, called a CM-point by $K$, which is fixed under all transformations in $\Phi(\varphi(K))$. They include the elliptic points, in which case the field $K$ is either $\mathbb{Q}(\sqrt{-1})$ or $\mathbb{Q}(\sqrt{-3})$.

A CM-point $P \in \mathcal{H}$ will be called special (SCM) if it is the fixed point of some fractional linear transformation $\gamma \in(\Phi \circ \varphi)\left(\mathcal{O}_{K}\right)$ of determinant equal to 6 , the discriminant of the algebra. Here $\mathcal{O}_{K}$ stands for the ring of integers of $K$.

The theory of canonical models tells us that the curve associated to $\Gamma_{6}$ has a canonical model $\left(X_{6}, j_{6}\right)$ which is defined over $\mathbb{Q}$. Hence there exists a one to one mapping (cf. section 2)

$$
j_{6}: \Gamma_{6} \backslash \mathcal{H} \rightarrow X_{6}(\mathbb{C}) .
$$

CM-points are relevant since their coordinates $j_{6}(P)$ generate class fields over imaginary quadratic fields.

A fundamental domain for the action of $\Gamma_{6}$ in $\mathcal{H}$, together with its elliptic points and SCM-points, was computed in [1]. This information is collected in table 1 and figure 1.

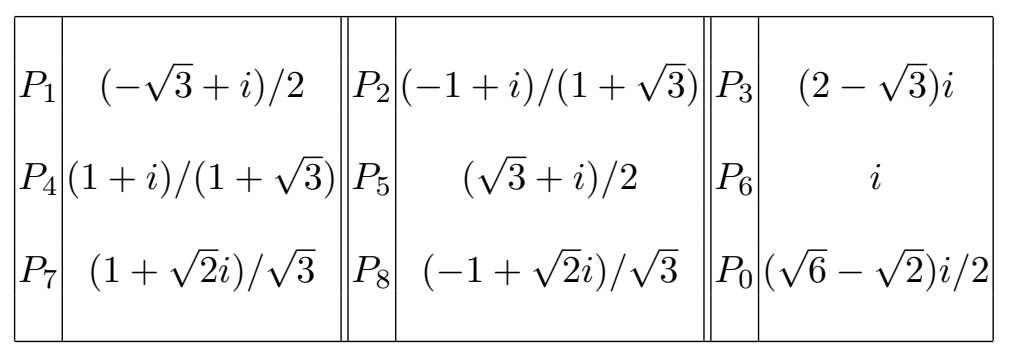

TABLE 1. Vertices of a fundamental domain for $X_{6}$ and SCM-points

Let $N\left(\mathcal{O}_{6}\right)$ be the normalizer of $\mathcal{O}_{6}$ in $\mathrm{H}_{6}$. The elements of $N\left(\mathcal{O}_{6}\right)$ of positive reduced norm define a subgroup whose image in $\mathbf{G L}^{+}(2, \mathbb{R})$ will be denoted by $\Gamma_{6}^{+}$. The group $\Gamma_{6}$ is contained in $\Gamma_{6}^{+}$as a normal subgroup and the quotient $\bar{\Gamma}_{6}^{+} / \bar{\Gamma}_{6}$ is isomorphic to $(\mathbb{Z} / 2 \mathbb{Z})^{2}$. The classes of this quotient are represented by elements $w_{d} \in \mathcal{O}_{6}$ of norm $d$ dividing 6 . We can take $w_{2}:=1+\mathrm{J}, w_{3}:=(-3-\mathrm{I}-3 \mathrm{~J}+\mathrm{IJ}) / 2$ and $w_{6}:=w_{2} w_{3}=-3 \mathrm{~J}+\mathrm{IJ}$. They define involutions of the curve $X_{6}$, denoted $\omega_{d}$

Together with the curve $X_{6}$, we consider its quotients $X_{6}^{(d)}:=X_{6} /\left\langle\omega_{d}\right\rangle$ and $X_{6}^{+}:=X_{6} /\left\langle\omega_{d}: d \mid 6\right\rangle$. All these curves fit in a diagram of Galois covers of degree 


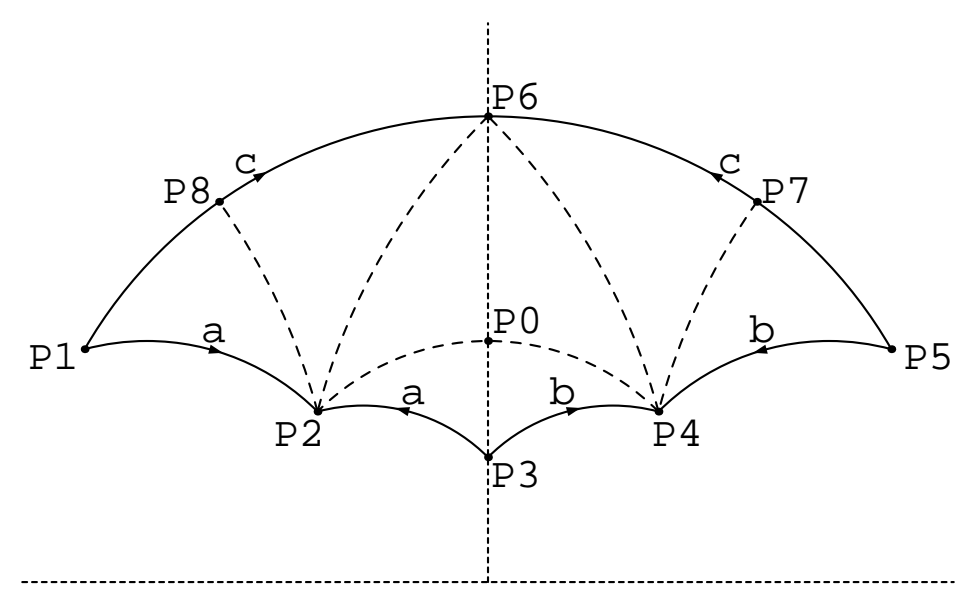

Figure 1. Fundamental domain for $X_{6}$

two:

$$
\begin{array}{ccc} 
& X_{6} \\
\swarrow & \downarrow & \searrow \\
X_{6}^{(2)} & X_{6}^{(3)} & X_{6}^{(6)} \\
\searrow & \downarrow & \swarrow \\
& X_{6}^{+} . &
\end{array}
$$

Figures 2 and 3 show fundamental domains for the genus zero curves $X_{6}^{(2)}, X_{6}^{(3)}$, $X_{6}^{(6)}, X_{6}^{+}$.
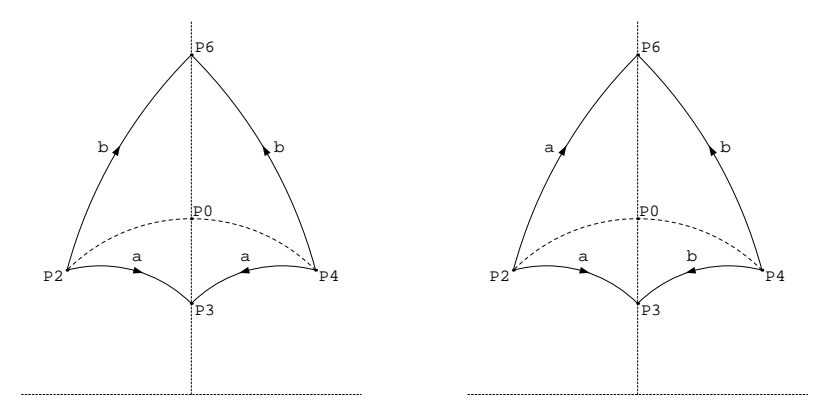

Figure 2. Fundamental domains for $\Gamma_{6}^{(2)}$ and $\Gamma_{6}^{(3)}$

The above fundamental domains exhibit particular symmetries. We use some of them to cut fundamental half-domains, that is, half-domains containing all the vertices of a fundamental domain exactly once. Table 2 lists axes of symmetries and polygons defining fundamental half-domains for the curves $X_{6}, X_{6}^{(2)}, X_{6}^{(3)}$, 

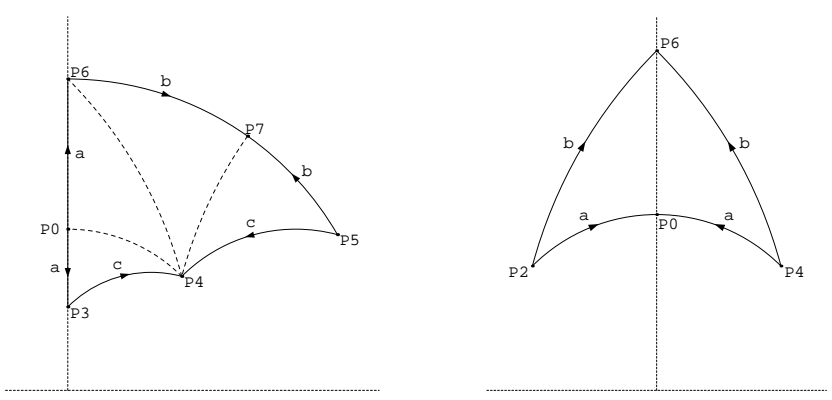

FiguRE 3. Fundamental domains for $\Gamma_{6}^{(6)}$ and $\Gamma_{6}^{+}$

$X_{6}^{(6)}, X_{6}^{+}$. The results displayed in table 3 show the angles at the different vertices of the polygons defining the corresponding fundamental half-domains.

\begin{tabular}{|c|c|c|}
\hline curve & axis of symmetry & polygons \\
\hline$X_{6}$ & $\left(P_{2}, P_{6}, P_{4}\right)$ & {$\left[P_{2}, P_{3}, P_{4}, P_{6}\right]$} \\
$X_{6}^{(2)}$ & $\left(P_{3}, P_{6}\right)$ & {$\left[P_{3}, P_{4}, P_{6}\right]$} \\
$X_{6}^{(3)}$ & $\left(P_{2}, P_{4}\right)$ & {$\left[P_{2}, P_{4}, P_{6}\right]$} \\
$X_{6}^{(6)}$ & $\left(P_{0}, P_{4}, P_{7}\right)$ & {$\left[P_{0}, P_{4}, P_{7}, P_{6}\right]$} \\
$X_{6}^{+}$ & $\left(P_{0}, P_{6}\right)$ & {$\left[P_{0}, P_{4}, P_{6}\right]$} \\
\hline
\end{tabular}

TABLE 2. Fundamental half-domains

\section{CANONical models}

Since the curves we are dealing with are all of genus zero, there exist automorphic functions on $\mathcal{H}, t_{6}, t_{6}^{(2)}, t_{6}^{(3)}, t_{6}^{(6)}, t_{6}^{+}$, uniquely defined by imposing their values at three vertices of the fundamental half-domains and such that they

\begin{tabular}{|c|c|c|c|c|c|c|}
\hline & $P_{0}$ & $P_{2}$ & $P_{3}$ & $P_{4}$ & $P_{6}$ & $P_{7}$ \\
\hline$X_{6}$ & $*$ & $\pi / 3$ & $\pi / 2$ & $\pi / 3$ & $\pi / 2$ & $*$ \\
$X_{6}^{(2)}$ & $*$ & $*$ & $\pi / 4$ & $\pi / 3$ & $\pi / 4$ & $*$ \\
$X_{6}^{(3)}$ & $*$ & $\pi / 6$ & $*$ & $\pi / 6$ & $\pi / 2$ & $*$ \\
$X_{6}^{(6)}$ & $\pi / 2$ & $*$ & $*$ & $\pi / 3$ & $\pi / 2$ & $\pi / 2$ \\
$X_{6}^{+}$ & $\pi / 2$ & $*$ & $*$ & $\pi / 6$ & $\pi / 4$ & $*$ \\
\hline
\end{tabular}

TABlE 3. Angles at the vertices of fundamental half-domains 


\begin{tabular}{|c|c|c|c|c|c|c|}
\hline$t$ & $P_{0}$ & $P_{2}$ & $P_{3}$ & $P_{4}$ & $P_{6}$ & $P_{7}$ \\
\hline$t_{6}$ & $*$ & $a$ & 0 & 1 & $\infty$ & $*$ \\
$t_{6}^{(2)}$ & $*$ & $*$ & 0 & 1 & $\infty$ & $*$ \\
$t_{6}^{(3)}$ & $*$ & 0 & $*$ & 1 & $\infty$ & $*$ \\
$t_{6}^{(6)}$ & 0 & $*$ & $*$ & 1 & $\infty$ & $b$ \\
$t_{6}^{+}$ & 0 & $*$ & $*$ & 1 & $\infty$ & $*$ \\
\hline
\end{tabular}

TABLE 4. Values of the uniformizing functions at the vertices

uniformize the curves $X_{6}, X_{6}^{(2)}, X_{6}^{(3)}, X_{6}^{(6)}, X_{6}^{+}$. We choose the functions in accordance with table 4 .

Proposition 2.1. The uniformizing functions defined in table 4 fulfil the following algebraic relations:

(1) $4 t_{6}^{+} t_{6}^{(2)}=\left(t_{6}^{(2)}+1\right)^{2}$.

(3) $4 t_{6}^{(2)}\left(2 t_{6}^{(3)}-1\right)^{2}=\left(t_{6}^{(2)}+1\right)^{2}$.

(2) $t_{6}^{+}=\left(2 t_{6}^{(3)}-1\right)^{2}$.

(5) $4 t_{6} t_{6}^{(3)}=\left(t_{6}+1\right)^{2}$.

(4) $t_{6}^{2}=t_{6}^{(2)}$.

(7) $2 t_{6} t_{6}^{(6)}=i\left(t_{6}-i\right)^{2}$.

(6) $t_{6}^{+}+t_{6}^{(6)}\left(t_{6}^{(6)}-2\right)=0$.

(9) $\left(t_{6}^{(2)}+1\right)^{2}+4 t_{6}^{(2)} t_{6}^{(6)}\left(t_{6}^{(6)}-2\right)=0$.

(8) $4 t_{6}^{2} t_{6}^{+}=\left(t_{6}^{2}+1\right)^{2}$.

Moreover, we have the following values for the functions:
(11) $t_{6}^{(2)}\left(P_{0}\right)=-1$.
(12) $t_{6}^{(3)}\left(P_{0}\right)=\frac{1}{2}$
$(13) b=t_{6}^{(6)}\left(P_{7}\right)=2$.
(14) $a=t_{6}\left(P_{2}\right)=-1$
(15) $t_{6}\left(P_{0}\right)=i$.
(16) $t_{6}\left(P_{7}\right)=-i$

For a non-constant smooth function $f(z)$, let $D(f, z)$ be its derivative. The automorphic derivative of $f$ is defined as

$$
D a(f, z)=\frac{2 D(f, z) D^{3}(f, z)-3 D^{2}(f, z)^{2}}{D(f, z)^{4}} .
$$

For a homographic transformation $\gamma(z)=\frac{a z+b}{c z+d}, \gamma=\left[\begin{array}{ll}a & b \\ c & d\end{array}\right] \in \mathbf{G L}(2, \mathbb{C})$, we have $D a(\gamma, z)=0$. Conversely, for a non-constant smooth function $\gamma(z)$, the relation $D a(\gamma, z)=0$ implies that $\gamma(z)=\frac{a z+b}{c z+d}$, for some $\gamma=\left[\begin{array}{ll}a & b \\ c & d\end{array}\right] \in \mathbf{M}(2, \mathbb{C})$.

The automorphic derivative $D a(f, z)$ of a $\Gamma$-automorphic function $f(z)$ is again a $\Gamma$-automorphic function. That is to say, for every $\gamma \in \Gamma, z \in \mathcal{H}$ the following equality holds: $D a(f, \gamma(z))=D a(f, z)$. 
Proposition 2.2. The functions defined by table 4 satisfy the differential equations $D a(t, z)+R(t)=0$, where the rational functions $R(t)$ are listed in table 5 .

Theorem 2.3. The functions $j_{6}^{+}, j_{6}^{(2)}, j_{6}^{(3)}, j_{6}^{(6)}: \mathcal{H} \rightarrow \mathbf{P}^{1}(\mathbb{C})$ given by

$$
\begin{aligned}
& j_{6}^{+}:=t_{6}^{+}, \quad j_{6}^{(2)}:=u_{2}:=\sqrt{-1} \frac{1+t_{6}^{(2)}}{1-t_{6}^{(2)}}, \\
& j_{6}^{(3)}:=u_{3}:=\sqrt{-3}\left(1-2 t_{6}^{(3)}\right), \quad j_{6}^{(6)}:=u_{6}:=\sqrt{-3}\left(1-t_{6}^{(6)}\right),
\end{aligned}
$$

and the function $j_{6}: \mathcal{H} \rightarrow \mathbf{P}^{2}(\mathbb{C})$ given in homogeneous coordinates by

$$
j_{6}:=\left(u_{3}: u_{6}: 1\right)=\left(u_{2}: 1: 1 / u_{6}\right)=\left(1: 1 / u_{2}: 1 / u_{3}\right)
$$

define canonical models over $\mathbb{Q}$ of the curves $X_{6}^{+}, X_{6}^{(2)}, X_{6}^{(3)}, X_{6}^{(6)}, X_{6}$, respectively. For the last one, we have

$$
\mathbb{Q}\left(X_{6}\right)=\mathbb{Q}\left(j_{6}\right)=\mathbb{Q}\left(u_{3}, u_{6}\right)=\mathbb{Q}\left(u_{2}, u_{6}\right)=\mathbb{Q}\left(u_{2}, u_{3}\right) .
$$

These functions fulfil the following algebraic relations:

$$
u_{3}^{2}+u_{6}^{2}+3=0, \quad u_{6} u_{2}=u_{3} .
$$

For the proofs of propositions 2.1, 2.2 and theorem 2.3 we refer the reader to $[6]$.

\section{UNIFORMIZING FUNCTIONS AND THETA CONSTANTS}

We consider the alternating bilinear form

$$
\mathrm{T}(\alpha, \beta):=\operatorname{Tr}(\mu \alpha \bar{\beta}), \quad \alpha, \beta \in \mathrm{H}_{6},
$$

where $\mu:=-3 \mathrm{~J}+\mathrm{IJ}$ is such that $\mu^{2}=-6$. The form $\mathrm{T}$ is integer valued over $\mathcal{O}_{6}$. The matrices

$$
\eta_{1}=1_{2}, \eta_{2}=\left[\begin{array}{cc}
\frac{-1+\sqrt{3}}{2} & \frac{-1+\sqrt{3}}{2} \\
\frac{1+\sqrt{3}}{2} & \frac{-1-\sqrt{3}}{2}
\end{array}\right], \eta_{3}=\left[\begin{array}{cc}
0 & -1 \\
1 & 0
\end{array}\right], \eta_{4}=\left[\begin{array}{cc}
-1+\sqrt{3} & 0 \\
0 & -1-\sqrt{3}
\end{array}\right]
$$

define a symplectic basis in $\Phi\left(\mathcal{O}_{6}\right)$ with respect to the bilinear form

$$
E(\Phi(\alpha), \Phi(\beta)):=\frac{1}{6} \mathrm{~T}(\alpha, \beta)
$$

(note that 6 equals the positive square root of the Pfaffian of $\mathrm{T}$ ). Therefore, for a given point $z \in \mathcal{H}$

$$
\Phi\left(\mathcal{O}_{6}\right)\left[\begin{array}{l}
z \\
1
\end{array}\right]=\mathbb{Z}\left[\eta_{1}(z), \eta_{2}(z), \eta_{3}(z), \eta_{4}(z)\right]
$$




\begin{tabular}{|c|c|c|c|}
\hline Curve & 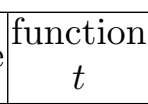 & $\begin{array}{c}\text { vertices } \\
\text { angles }\end{array}$ & $-D a(t, z)$ \\
\hline$X_{6}$ & $t_{6}$ & $\begin{array}{c}{\left[P_{2}, P_{3}, P_{4}, P_{6}\right]} \\
{[\pi / 3, \pi / 2, \pi / 3, \pi / 2]}\end{array}$ & $\frac{27 t^{4}+74 t^{2}+27}{36 t^{2}\left(t^{2}-1\right)^{2}}$ \\
\hline$X_{6}^{(2)}$ & $t_{6}^{(2)}$ & $\begin{array}{c}{\left[P_{3}, P_{4}, P_{6}\right]} \\
{[\pi / 4, \pi / 3, \pi / 4]}\end{array}$ & $\frac{135 t^{2}-142 t+135}{144 t^{2}(t-1)^{2}}$ \\
\hline$X_{6}^{(3)}$ & $t_{6}^{(3)}$ & $\begin{array}{c}{\left[P_{2}, P_{4}, P_{6}\right]} \\
{[\pi / 6, \pi / 6, \pi / 2]}\end{array}$ & $\frac{27 t^{2}-27 t+35}{36 t^{2}(t-1)^{2}}$ \\
\hline$X_{6}^{(6)}$ & $t_{6}^{(6)}$ & $\begin{array}{c}{\left[P_{0}, P_{4}, P_{7}, P_{6}\right]} \\
{[\pi / 2, \pi / 3, \pi / 2, \pi / 2]}\end{array}$ & $\frac{27 t^{4}-108 t^{3}+211 t^{2}-206 t+108}{36 t^{2}\left(t^{2}-3 t+2\right)^{2}}$ \\
\hline$X_{6}^{+}$ & $t_{6}^{+}$ & $\begin{array}{c}{\left[P_{0}, P_{4}, P_{6}\right]} \\
{[\pi / 2, \pi / 6, \pi / 4]}\end{array}$ & $\frac{135 t^{2}-103 t+108}{144 t^{2}(t-1)^{2}}$ \\
\hline
\end{tabular}

TABLE 5. Automorphic derivatives of the functions

We define two period matrices by $\Omega_{1}(z)=\left[\eta_{1}(z), \eta_{2}(z)\right], \Omega_{2}(z)=\left[\eta_{3}(z), \eta_{4}(z)\right]$. Let $\varepsilon$ be the embedding of the Poincaré upper half-plane $\mathcal{H}$ into the Siegel upper half-space $\mathcal{H}_{2}$ of degree 2 defined by

$$
\begin{aligned}
\varepsilon: \mathcal{H} & \rightarrow \mathcal{H}_{2} \\
& z \mapsto \Omega_{1}(z)^{-1} \cdot \Omega_{2}(z),
\end{aligned}
$$

that is

$$
\varepsilon(z)=\frac{1}{1-\sqrt{3}-2 \sqrt{3} z+(1+\sqrt{3}) z^{2}}\left[\begin{array}{cc}
1+\sqrt{3}-2 \sqrt{3} z+(1-\sqrt{3}) z^{2} & 2\left(1+z^{2}\right) \\
2\left(1+z^{2}\right) & -4 \sqrt{3} z
\end{array}\right] .
$$

Henceforth we let $\mathbf{S p}(4, \mathbb{R})$ be the group of the symplectic $(4 \times 4)$-matrices.

We let $\Gamma_{6}$ and the transformations $\Phi\left(w_{d}\right), d \mid 6$, act on $\Phi\left(\mathcal{O}_{6}\right)$ by multiplication on the right. Since the action of $\Gamma_{6}$ preserves the alternating form $E$, and the elements $\Phi\left(w_{d}\right)$ act as symplectic similarities, we obtain two embeddings

$$
\Psi: \Gamma_{6} \rightarrow \mathbf{S p}(4, \mathbb{Z}), \quad \Psi^{+}: \Gamma_{6}^{+} \rightarrow \mathbf{S p}(4, \mathbb{R}),
$$

which, moreover, are compatible with $\varepsilon$. Hence, there exist well-defined mappings

$$
\Gamma_{6} \backslash \mathcal{H} \rightarrow \mathbf{S p}(4, \mathbb{Z}) \backslash \mathcal{H}_{2}, \quad \Gamma_{6}^{+} \backslash \mathcal{H} \rightarrow \operatorname{Sp}_{6}^{+}(4, \mathbb{Z}) \backslash \mathcal{H}_{2},
$$

where $\operatorname{Sp}_{6}^{+}(4, \mathbb{Z})$ denotes the subgroup of $\mathbf{S p}(4, \mathbb{R})$ generated by $\mathbf{S p}(4, \mathbb{Z})$ and $\Psi^{+}\left(\Gamma_{6}^{+}\right)$. 
Let $x_{1}, x_{2}, \ldots, x_{6}$ be the roots of a sextic

$$
a_{6} X^{6}+a_{5} X^{5}+\cdots+a_{0}, \quad a_{i} \in \mathbb{C} .
$$

Let us write $(i, j)$ as an abridged notation for $x_{i}-x_{j}$ and define, in accordance with [12] and [7], the following symmetric functions of the roots:

$$
\begin{aligned}
I_{2}(a) & =a_{6}^{2} \sum_{(15)}(12)^{2}(34)^{2}(56)^{2}, \\
I_{4}(a) & =a_{6}^{4} \sum_{(10)}(12)^{2}(23)^{2}(31)^{2}(45)^{2}(56)^{2}(64)^{2}, \\
I_{6}(a) & =a_{6}^{6} \sum_{(60)}(12)^{2}(23)^{2}(31)^{2}(45)^{2}(56)^{2}(64)^{2}(14)^{2}(25)^{2}(36)^{2}, \\
I_{10}(a) & =a_{6}^{10} \prod_{i<j}(i j)^{2},
\end{aligned}
$$

where $a=\left(a_{0}, \ldots, a_{6}\right)$. Then, the projective Igusa invariants of the sextic are defined as

$$
\begin{aligned}
& J_{2}=2^{-3} I_{2}, \quad J_{4}=2^{-5} 3^{-1}\left(4 J_{2}^{2}-I_{4}\right), \\
& J_{6}=2^{-6} 3^{-2}\left(8 J_{2}^{3}-160 J_{2} J_{4}-I_{6}\right), \\
& J_{8}=2^{-2}\left(J_{2} J_{6}-J_{4}^{2}\right), \quad J_{10}=2^{-12} I_{10},
\end{aligned}
$$

and the absolute Igusa invariants as

$$
i_{1}=\frac{I_{2}^{5}}{I_{10}}, \quad i_{2}=\frac{I_{2}^{3} I_{4}}{I_{10}}, \quad i_{3}=\frac{I_{2}^{2} I_{6}}{I_{10}} .
$$

As is well known, the points of $\operatorname{Proj} \mathbb{C}\left[I_{2}, I_{4}, I_{8}, I_{10}\right]$ not on $I_{10}=0$ form the variety $\mathcal{M}_{2}$ of moduli of genus 2 curves.

We recall that, using theta constants, the Igusa invariants can be computed from the period matrix of the non-singular genus 2 curve with hyperelliptic model

$$
C: Y^{2}=a_{6} X^{6}+a_{5} X^{5}+\cdots+a_{0} .
$$

We consider the Riemann theta function

$$
\vartheta(z ; Z)=\sum_{n \in \mathbb{Z}^{2}} \exp \left(\pi i^{t} n \cdot Z \cdot n+2 \pi i^{t} n \cdot z\right), \quad z \in \mathbb{C}^{2}, Z \in \mathcal{H}_{2} .
$$

Given a pair $(r, s)$ of column vectors, $r, s \in\{0,1 / 2\}^{2}$, let $c={ }^{t}[r, s]$. The theta function with half-integral characteristic $c$ is defined, for $z \in \mathbb{C}^{2}, Z \in \mathcal{H}_{2}$, by

$$
\vartheta[c](z ; Z)=\exp \left(\pi i^{t} r \cdot Z \cdot r+2 \pi i^{t} r \cdot(z+s)\right) \vartheta(z+Z \cdot r+s ; Z) .
$$

It is an even or odd function of $z$ according to the parity of $c$ :

$$
\vartheta[c](z ; Z) \text { is }\left\{\begin{array} { l } 
{ \text { even } } \\
{ \text { odd } }
\end{array} \Longleftrightarrow 4 ^ { t } r \cdot s \equiv \left\{\begin{array}{ll}
0 & (\bmod 2) . \\
1 &
\end{array}\right.\right.
$$

For simplicity, we denote by $\vartheta_{i}(Z)=\vartheta\left[c_{i}\right](0 ; Z)$ the ten thetaconstants (Thetanullwerte) which correspond to the ten even characteristics (in a given order). 
There are Siegel modular forms $h_{4}, h_{10}, h_{12}, h_{16}$, of the respective weights, defined in terms of theta constants, such that

$$
I_{2}=\frac{h_{12}}{h_{10}}, \quad I_{4}=h_{4}, \quad I_{6}=\frac{h_{16}}{h_{10}}, \quad I_{10}=h_{10} .
$$

In fact,

$$
h_{4}=\sum_{i=1}^{10} \vartheta_{i}^{8}, \quad h_{10}=\prod_{i=1}^{10} \vartheta_{i}^{2} .
$$

The expressions for $h_{12}, h_{16}$ are more involved. They can be found in [7] and [9].

The following proposition shows how the function $t_{6}^{+}$can be obtained by restriction of Siegel automorphic functions.

Theorem 3.1. $\quad$ (i) We have

$$
\begin{aligned}
t_{6}^{+}(z) & =1+\frac{\left(J_{2}^{2}(\varepsilon(z))-24 J_{4}(\varepsilon(z))\right)^{5}}{2^{16} 3^{13} J_{10}^{2}(\varepsilon(z))} \\
& =-\frac{1}{3^{3}} \frac{5 J_{2}^{3}(\varepsilon(z))+12 J_{2}(\varepsilon(z)) J_{4}(\varepsilon(z))-2376 J_{6}(\varepsilon(z))}{J_{2}^{3}(\varepsilon(z))-36 J_{2}(\varepsilon(z)) J_{4}(\varepsilon(z))+216 J_{6}(\varepsilon(z))},
\end{aligned}
$$

for every $z \in \mathcal{H}$.

(ii) There exist homogeneous polynomials $A, B \in \overline{\mathbb{Q}}\left[T_{1}, \ldots, T_{10}\right]$ of degree 40, respectively $C, D \in \overline{\mathbb{Q}}\left[T_{1}, \ldots, T_{10}\right]$ of degree 12, such that

$t_{6}^{+}(z)=\frac{A\left(\vartheta_{1}(\varepsilon(z)), \ldots, \vartheta_{10}(\varepsilon(z))\right)}{B\left(\vartheta_{1}(\varepsilon(z)), \cdots, \vartheta_{10}(\varepsilon(z))\right)}=\frac{C\left(\vartheta_{1}(\varepsilon(z)), \ldots, \vartheta_{10}(\varepsilon(z))\right)}{D\left(\vartheta_{1}(\varepsilon(z)), \cdots, \vartheta_{10}(\varepsilon(z))\right)}$,

for every $z \in \mathcal{H}$.

Proof. (Sketch) Expression (i) follows from the work of Hashimoto-Murabayashi [11], Baba-Granath [2] and Rotger [15], [16]. In fact, Hashimoto-Murabayashi gave a parametric family of curves of genus 2 whose jacobian has quaternionic multiplication by $\mathcal{O}_{6}$. Using their result, Baba-Granath expressed a Hauptmodul for $\Gamma_{6}^{+}$in terms of Igusa invariants. Since Rotger proved that the quaternionic locus in Igusa's threefold $\mathcal{A}_{2}$ corresponding to $D=6$ is irreducible, a computation of the Baba-Granath function at three points allows the identification of our function $t_{6}^{+}$.

Examples 3.2. The absolute Igusa invariants at $\varepsilon\left(P_{0}\right)$ and $\varepsilon\left(P_{7}\right)$ coincide. They were obtained by Bayer-Guàrdia [4] as

$$
i_{1}=-\frac{2 \cdot 11^{5}}{3}, \quad i_{2}=2 \cdot 3^{2} \cdot 11^{3}, \quad i_{3}=2 \cdot 3 \cdot 7 \cdot 11^{2},
$$


from the genus 2 curves

$$
\begin{gathered}
C_{1}: Y^{2}=X^{5}-2 i \sqrt{2} X^{4}-\frac{11}{3} X^{3}+2 i \sqrt{2} X^{2}+X \\
C_{2}: Y^{2}=X^{5}+2 \sqrt[4]{-5+2 \sqrt{6}} X^{4}-\frac{10}{3} i \sqrt{2} X^{3}+2 i \sqrt[4]{-5-2 \sqrt{6}} X^{2}+X .
\end{gathered}
$$

The periods of these curves are $\varepsilon\left(P_{0}\right)$ and $\varepsilon\left(P_{7}\right)$, respectively, and their jacobians have quaternionic multiplication by $\mathcal{O}_{6}$, so that these are fake elliptic curves in the sense of Serre. A direct computation using the second terms in theorem 3.1(i) recovers the values $t^{+}\left(P_{0}\right)=t^{+}\left(P_{7}\right)=0$, given in table 4 .

\section{Local parameters}

To obtain explicit expansions of the uniformizing functions around the elliptic points and around the CM-points we make a first choice of local parameters.

Let $\Gamma \subseteq \mathbf{G L}^{+}(2, \mathbb{R})$ be a Fuchsian group projected in $\bar{\Gamma} \subseteq \mathbf{P S L}(2, \mathbb{R})$. Let $\bar{\Gamma}_{P}$ denote the isotropy group at a point $P \in \mathcal{H}$ of order $e=\# \bar{\Gamma}_{P}$. We can expand each $\bar{\Gamma}_{P}$-automorphic function $t$ around the point $P$ as a power series $T$ in the variable $h(z)=k \frac{z-P}{z-\bar{P}}$. If $t(z)=T(h(z))=\sum_{n=n_{0}}^{\infty} a_{n} h(z)^{n}$, we shall have $T(h(z))=T\left(\zeta^{2} h(z)\right)$, where $\zeta$ is a $2 e$-primitive root of unity. Thus $a_{n}=0$ unless $n \equiv 0(\bmod e)$.

Definition 4.1. A local parameter at a point $P \in \mathcal{H}$ for the $\bar{\Gamma}_{P}$-action is any function

$$
q(z):=\left(k \frac{z-P}{z-\bar{P}}\right)^{e}
$$

where $e=\# \bar{\Gamma}_{P}$ is the order of the isotropy group at $P$ and $k \in \mathbb{C}-\{0\}$ is a constant. The local parameter $q(z)$ is said to be adapted to a non-constant $\Gamma$-automorphic function $t(z)=\sum_{n=m}^{\infty} a_{n e} q(z)^{n}$ if, moreover, $a_{r e}=1$ in the case that $t-a_{0}$ has a zero of order $r e$ at $z=P$, or $a_{-r e}=1$ if $t$ has a pole of order $r e$ at $z=P$. The corresponding adapted local constant $k$ will be denoted by $k(P, \Gamma, t)$ (occasionally by $k_{P}$ when no confusion can arise). Note that the adapted local constants are defined up to the product by an $e$-th root of unity.

Let us consider the hypergeometric function defined by the series

$$
F(a, b, c ; w)=\sum_{n=0}^{\infty} \frac{(a)_{n}(b)_{n}}{(c)_{n}} \frac{w^{n}}{n !}, \quad(a)_{n}:=a(a+1) \ldots(a+n-1)=\frac{\Gamma(a+n)}{\Gamma(a)},
$$


which converges for $|w|<1$. Assume that $c \neq 1$. The Schwarzian function

$$
z=s(a, b, c ; w):=\frac{w^{1-c} F(a-c+1, b-c+1,2-c ; w)}{F(a, b, c ; w)}
$$

maps the upper half $w$-plane $\mathcal{H}$ onto a triangle in the $z$-plane whose vertices can be expressed in terms of the Euler's $\Gamma$-function in the following way:

$$
\begin{aligned}
& s(a, b, c ; 0)=0, \\
& s(a, b, c ; 1)=\frac{\Gamma(c-a) \Gamma(c-b) \Gamma(2-c)}{\Gamma(c) \Gamma(1-b) \Gamma(1-a)}, \\
& s(a, b, c ; \infty)=e^{\pi i(1-c)} \frac{\Gamma(c-a) \Gamma(b) \Gamma(2-c)}{\Gamma(c) \Gamma(b-c+1) \Gamma(1-a)} .
\end{aligned}
$$

The internal angles at these vertices are $\alpha \pi, \beta \pi, \gamma \pi$, where

$$
\alpha=1-c \neq 0, \quad \beta=c-a-b, \quad \gamma=b-a .
$$

By comparing the triangle $[s(0), s(1), s(\infty)]$ with the triangles we have used to define the functions $t_{6}^{+}, t_{6}^{(2)}$, and $t_{6}^{(3)}$, we obtain the corresponding adapted local constants $k_{P}$ at the elliptic points and SCM-points $P$. They are listed in table 6 .

To compute the local parameters for the two remaining uniformizing functions, we use the following result, which relates adapted local constants in some particular situations.

Proposition 4.2. Let $t$ be a non-constant automorphic function for the action of a Fuchsian group $\Gamma \subseteq \mathbf{G L}^{+}(2, \mathbb{R})$, and $P \in \mathcal{H}$ a point of order $e \geq 1$ for $\bar{\Gamma}_{P}$.

(i) For each $w=\left[\begin{array}{l}a \\ c \\ c d\end{array}\right] \in \Gamma$, the constants $k(P, \Gamma, t), k(w(P), \Gamma, t)$ are related by

$$
k(w(P), \Gamma, t)^{e r}=k(P, \Gamma, t)^{e r}\left(\frac{c P+d}{c \bar{P}+d}\right)^{e r},
$$

where the value of $r$ is given in definition 4.1.

(ii) Assume that $t$ has a pole of order re at $P$. Then the constants $k(P, \Gamma, t)$ and $k\left(P, \Gamma, \frac{1}{1-t}\right)$ are related by

$$
k(P, \Gamma, t)^{r e}=-k\left(P, \Gamma, \frac{1}{1-t}\right)^{r e}
$$




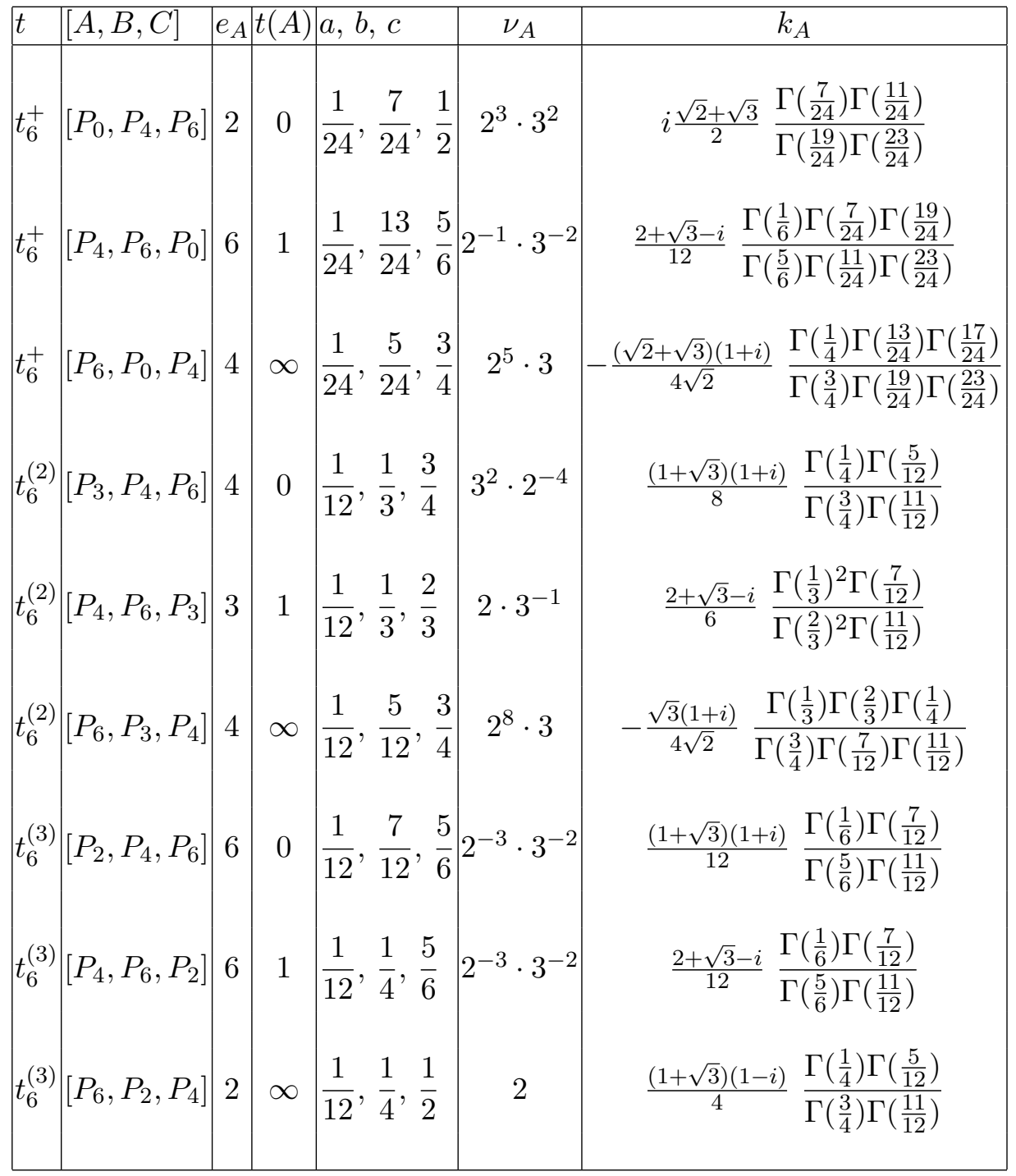

TABLE 6. Adapted local constants for the triangle functions

(iii) Assume that $t(P)=v, v \neq 0, \infty$. Then the constants $k(P, \Gamma, t)$ and $k\left(P, \Gamma, 1-\frac{v}{t}\right)$ are related by

$$
k(P, \Gamma, t)^{r e}=v k\left(P, \Gamma, 1-\frac{v}{t}\right)^{r e}
$$




\begin{tabular}{|c|c|c|c|c|c|}
\hline$t$ & $P$ & $e_{P}$ & $t(P)$ & $\nu_{P}$ & $k_{P}$ \\
\hline$t_{6}^{(6)}$ & $P_{0}$ & 2 & 0 & $2^{2} \cdot 3^{2}$ & $i \frac{\sqrt{2}+\sqrt{3}}{2 \sqrt{2}} \frac{\Gamma\left(\frac{7}{24}\right) \Gamma\left(\frac{11}{24}\right)}{\Gamma\left(\frac{19}{24}\right) \Gamma\left(\frac{23}{24}\right)}$ \\
\hline$t_{6}^{(6)}$ & $P_{4}$ & 3 & 1 & $3^{-1}$ & $\frac{2+\sqrt{3}+i}{12} \frac{\Gamma\left(\frac{1}{6}\right) \Gamma\left(\frac{7}{24}\right) \Gamma\left(\frac{19}{24}\right)}{\Gamma\left(\frac{5}{6}\right) \Gamma\left(\frac{11}{24}\right) \Gamma\left(\frac{23}{24}\right)}$ \\
\hline$t_{6}^{(6)}$ & $P_{7}$ & 2 & 2 & $2^{2} \cdot 3^{2}$ & $\frac{(2 \sqrt{3}+3 \sqrt{2})(1-\sqrt{2} i)}{12} \frac{\Gamma\left(\frac{7}{24}\right) \Gamma\left(\frac{11}{24}\right)}{\Gamma\left(\frac{19}{24}\right) \Gamma\left(\frac{23}{24}\right)}$ \\
\hline$t_{6}^{(6)}$ & $P_{6}$ & 2 & $\infty$ & $2^{2}$ & $i \frac{\sqrt{2}+\sqrt{3}}{4} \frac{\Gamma\left(\frac{1}{4}\right) \Gamma\left(\frac{13}{24}\right) \Gamma\left(\frac{17}{24}\right)}{\Gamma\left(\frac{3}{4}\right) \Gamma\left(\frac{19}{24}\right) \Gamma\left(\frac{23}{24}\right)}$ \\
\hline$t_{6}$ & $P_{0}$ & 1 & $i$ & $2^{2} \cdot 3$ & $i \frac{\sqrt{2}+\sqrt{3}}{2} \frac{\Gamma\left(\frac{7}{24}\right) \Gamma\left(\frac{11}{24}\right)}{\Gamma\left(\frac{19}{24}\right) \Gamma\left(\frac{23}{24}\right)}$ \\
\hline$t_{6}$ & $P_{2}$ & 3 & -1 & $3^{-1}$ & $\frac{1+(2+\sqrt{3}) i}{6 \sqrt[3]{2}} \frac{\Gamma\left(\frac{1}{3}\right)^{2} \Gamma\left(\frac{7}{12}\right)}{\Gamma\left(\frac{2}{3}\right)^{2} \Gamma\left(\frac{11}{12}\right)}$ \\
\hline$t_{6}$ & $P_{3}$ & 2 & 0 & $3 \cdot 2^{-2}$ & $\frac{(1+\sqrt{3})(1+i)}{8} \frac{\Gamma\left(\frac{1}{4}\right) \Gamma\left(\frac{5}{12}\right)}{\Gamma\left(\frac{3}{4}\right) \Gamma\left(\frac{11}{12}\right)}$ \\
\hline$t_{6}$ & $P_{4}$ & 3 & 1 & $3^{-1}$ & $\frac{2+\sqrt{3}-i}{6 \sqrt[3]{2}} \frac{\Gamma\left(\frac{1}{3}\right)^{2} \Gamma\left(\frac{7}{12}\right)}{\Gamma\left(\frac{2}{3}\right)^{2} \Gamma\left(\frac{11}{12}\right)}$ \\
\hline$t_{6}$ & $P_{6}$ & 2 & $\infty$ & $2^{4}$ & $\frac{\sqrt{3}(1-i)}{4 \sqrt{2}} \frac{\Gamma\left(\frac{1}{3}\right) \Gamma\left(\frac{2}{3}\right) \Gamma\left(\frac{1}{4}\right)}{\Gamma\left(\frac{3}{4}\right) \Gamma\left(\frac{7}{12}\right) \Gamma\left(\frac{11}{12}\right)}$ \\
\hline
\end{tabular}

TABLE 7. Adapted local constants for the quadrilateral functions

Table 7 lists the local constants $k_{P}$ adapted to the quadrilateral uniformizing functions $t_{6}$ and $t_{6}^{(6)}$ in neighbourhoods of the vertices $P$ of fundamental halfdomains and, also, in a neighbourhood of the SCM-point $P_{0}$ for the function $t_{6}$.

In all the cases we are dealing with, it is $r=1$. By considering an adapted local parameter

$$
q_{P}(z)=\left(k_{P} \frac{z-P}{z-\bar{P}}\right)^{e_{P}}
$$


as a uniformizing variable in the neighbourhood of a point $P$, we obtain series expansions

$$
\begin{array}{ll}
t(z)=t(P)+\sum_{n=1}^{\infty} b_{n} q^{n}, & b_{n}:=a_{n e}, b_{1}=1, \text { if } t(P) \neq \infty \\
t(z)=\sum_{n=-1}^{\infty} b_{n} q^{n}, & b_{n}:=a_{n e}, b_{-1}=1, \text { if } t(P)=\infty
\end{array}
$$

\section{LOCAL EXPANSIONS}

We now normalize the uniformizing functions considered in the preceding sections.

Case $t(P) \neq \infty$. We consider expansions of the shape

$$
t(z)=\sum_{n=0}^{\infty} b_{n}^{\prime} \frac{q(z)^{n}}{(e n) !}, \quad b_{1}^{\prime}=e !
$$

and normalize the function $q$ by replacing $q$ by $\nu^{-1} q$, where the values of $\nu$ are listed in tables 6 and 7 . Then we define the factor $\mathfrak{n}_{v}=\nu e$ !, where $v=t(P)$, and normalize the generating function $t$ by $t\left(P, q_{P} ; z\right):=\mathfrak{n}_{v}^{-1} t(z)$ so that

$$
t\left(P, q_{P} ; z\right)=\sum_{n=0}^{\infty} c_{n} \frac{q_{P}(z)^{n}}{(e n) !}, \quad c_{1}=1, \quad q_{P}(z)=\frac{1}{\nu_{P}}\left(k_{P} \frac{z-P}{z-\bar{P}}\right)^{e_{P}},
$$

where the values of $e_{P}, k_{P}$ and $\nu_{P}$ are listed in tables 6 and 7 .

Case $t(P)=\infty$. We consider expansions of the shape

$$
t(z)=\sum_{n=-1}^{\infty} b_{n}^{\prime} \frac{q(z)^{n}}{(2 e(n+2)) !}, \quad b_{-1}^{\prime}=(2 e) !
$$

and normalize the function $q$ by replacing $q$ by $\nu^{-1} q$, where the values of $\nu$ are listed in tables 6 and 7 . Then we define the factor $\mathfrak{n}_{\infty}=\nu(2 e)$ ! and normalize the generating function $t$ by $t\left(P, q_{P} ; z\right):=\mathfrak{n}_{\infty}^{-1} t(z)$ so that

$$
t\left(P, q_{P} ; z\right)=\sum_{n=-1}^{\infty} c_{n} \frac{q_{P}(z)^{n}}{(2 e(n+2)) !}, \quad c_{-1}=1, \quad q_{P}(z)=\frac{1}{\nu_{P}}\left(k_{P} \frac{z-P}{z-\bar{P}}\right)^{e_{P}}
$$

where the values of $e_{P}, k_{P}$ and $\nu_{P}$ are listed in tables 6 and 7 .

Each generating function $t\left(P, q_{P} ; z\right)$ is a representative of the homothety class of the corresponding function $t$ which depends on the point $P$. The relations between them are compiled in table 8 .

As an example, we provide the starting coefficients for some series expansions associated to the complex uniformizing function $t_{6}$ of the curve $X_{6}$. 


\begin{tabular}{|c|c|c|c|c|c|}
\hline $\begin{array}{l}X \\
t\end{array}$ & $\begin{array}{c}X_{6}^{+} \\
t_{6}^{+} \\
\end{array}$ & $\begin{array}{c}X_{6}^{(2)} \\
t_{6}^{(2)} \\
\end{array}$ & $\begin{array}{c}X_{6}^{(3)} \\
t_{6}^{(3)} \\
\end{array}$ & $\begin{array}{c}X_{6}^{(6)} \\
t_{6}^{(6)} \\
\end{array}$ & $\begin{array}{l}X_{6} \\
t_{6} \\
\end{array}$ \\
\hline $\mathfrak{n}_{\infty}$ & $\begin{array}{c}3870720= \\
2^{12} \cdot 3^{3} \cdot 5 \cdot 7 \\
t_{6}^{+}\left(P_{6}, q_{P_{6}}\right)\end{array}$ & $\begin{array}{c}30965760= \\
2^{15} \cdot 3^{3} \cdot 5 \cdot 7 \\
t_{6}^{(2)}\left(P_{6}, q_{P_{6}}\right)\end{array}$ & $\begin{array}{l}48=2^{4} \cdot 3 \\
t_{6}^{(3)}\left(P_{6}, q_{P_{6}}\right)\end{array}$ & $\begin{array}{l}96=2^{5} \cdot 3 \\
t_{6}^{(6)}\left(P_{6}, q_{P_{6}}\right)\end{array}$ & $\begin{array}{l}384=2^{7} \cdot 3 \\
t_{6}\left(P_{6}, q_{P_{6}}\right)\end{array}$ \\
\hline $\mathfrak{n}_{0}$ & $\begin{array}{c}144=2^{4} \cdot 3^{2} \\
t_{6}^{+}\left(P_{0}, q_{P_{0}}\right)\end{array}$ & $\begin{array}{l}\frac{27}{2}=2^{-1} \cdot 3^{3} \\
t_{6}^{(2)}\left(P_{3}, q_{P_{3}}\right)\end{array}$ & $\begin{array}{c}10=2 \cdot 5 \\
t_{6}^{(3)}\left(P_{2}, q_{P_{2}}\right)\end{array}$ & $\begin{array}{l}72=2^{3} \cdot 3^{2} \\
t_{6}^{(6)}\left(P_{0}, q_{P_{0}}\right)\end{array}$ & $\begin{array}{l}\frac{3}{2}=2^{-1} \cdot 3 \\
t_{6}\left(P_{3}, q_{P_{3}}\right)\end{array}$ \\
\hline $\mathfrak{n}_{1}$ & $\begin{array}{l}40=2^{3} \cdot 5 \\
t_{6}^{+}\left(P_{4}, q_{P_{4}}\right)\end{array}$ & $\begin{array}{c}4=2^{2} \\
t_{6}^{(2)}\left(P_{4}, q_{P_{4}}\right)\end{array}$ & $\begin{array}{c}10=2 \cdot 5 \\
t_{6}^{(3)}\left(P_{4}, q_{P_{4}}\right)\end{array}$ & $t_{6}^{(6)}\left(\begin{array}{c}2 \\
\left(P_{4}, q_{P_{4}}\right)\end{array}\right.$ & $\begin{array}{c}2 \\
t_{6}\left(P_{4}, q_{P_{4}}\right)\end{array}$ \\
\hline $\mathfrak{n}_{2}$ & * & $\begin{array}{l}* \\
*\end{array}$ & $\begin{array}{l}* \\
*\end{array}$ & $\begin{array}{l}72=2^{3} \cdot 3^{2} \\
t_{6}^{(6)}\left(P_{7}, q_{P_{7}}\right)\end{array}$ & $\begin{array}{l}* \\
*\end{array}$ \\
\hline $\mathfrak{n}_{-1}$ & $\begin{array}{l}* \\
*\end{array}$ & $\begin{array}{l}* \\
*\end{array}$ & * & $\begin{array}{l}* \\
*\end{array}$ & $\begin{array}{c}2 \\
t_{6}\left(P_{2}, q_{P_{2}}\right)\end{array}$ \\
\hline $\mathfrak{n}_{i}$ & $\begin{array}{l}* \\
*\end{array}$ & $\begin{array}{l}* \\
*\end{array}$ & $\begin{array}{l}* \\
*\end{array}$ & $\begin{array}{l}* \\
*\end{array}$ & $\begin{array}{l}12=2^{2} \cdot 3 \\
t_{6}\left(P_{0}, q_{P_{0}}\right)\end{array}$ \\
\hline
\end{tabular}

TABLE 8. Local uniformizing functions $t\left(P, q_{P} ; z\right)=\mathfrak{n}_{t(P)}^{-1} \cdot t(z)$

Coefficients $c_{n}(1 \leq n \leq 10)$ of $t_{6}\left(P_{3}, q_{P_{3}} ; z\right)$ :

$$
\begin{aligned}
1 & =1 \\
0 & =0 \\
-48 & =-2^{4} \cdot 3 \\
0 & =0 \\
27504 & =2^{4} \cdot 3^{2} \cdot 191 \\
0 & =0 \\
-64498392 & =-2^{3} \cdot 3^{2} \cdot 7 \cdot 127973 \\
0 & =0 \\
436272183216 & =2^{4} \cdot 3^{4} \cdot 23 \cdot 229 \cdot 63913 \\
0 & =0
\end{aligned}
$$


Coefficients $c_{n}(0 \leq n \leq 10)$ of $t_{6}\left(P_{4}, q_{P_{4}} ; z\right)$ :

$$
\begin{aligned}
1 / 2 & =2^{-1} \\
1 & =1 \\
20 & =2^{2} \cdot 5 \\
1356 & =2^{2} \cdot 3 \cdot 113 \\
227040 & =2^{5} \cdot 3 \cdot 5 \cdot 11 \cdot 43 \\
74611380 & =2^{2} \cdot 3 \cdot 5 \cdot 1243523 \\
42574294080 & =2^{6} \cdot 3^{2} \cdot 5 \cdot 17 \cdot 19 \cdot 45767 \\
38683567274400 & =2^{5} \cdot 3^{2} \cdot 5^{2} \cdot 5372717677 \\
52554612744944640 & =2^{10} \cdot 3^{2} \cdot 5 \cdot 11 \cdot 23 \cdot 4507937111 \\
101782604056899960000 & =2^{6} \cdot 3^{4} \cdot 5^{4} \cdot 139 \cdot 226002762361 \\
270629344957362042528000 & =2^{8} \cdot 3^{4} \cdot 5^{3} \cdot 29 \cdot 16126171 \cdot 223259851
\end{aligned}
$$

Coefficients $c_{n}(0 \leq n \leq 10)$ of $t_{6}\left(P_{0}, q_{P_{0}} ; z\right)$ :

$$
\begin{aligned}
i / 12 & =-i \cdot(1+i)^{-4} \cdot 3^{-1} \\
1 & =1 \\
-12 i & =i \cdot(1+i)^{4} \cdot 3 \\
-226 & =-2 \cdot 113 \\
5664 i & =(1+i)^{10} \cdot 3 \cdot 59 \\
160728 & =2^{3} \cdot 3 \cdot 37 \cdot 181 \\
-5467296 i & =-(1+i)^{10} \cdot 3 \cdot 56951 \\
-211472208 & =-2^{4} \cdot 3^{5} \cdot 109 \cdot 499 \\
9193300992 i & =-i \cdot(1+i)^{20} \cdot 3^{2} \cdot 571 \cdot 1747 \\
445513958784 & =2^{7} \cdot 3^{3} \cdot 128910289 \\
-23734590202368 i & =-(1+i)^{18} \cdot 3^{4} \cdot 15919 \cdot 35951
\end{aligned}
$$


Coefficients $c_{n}(-1 \leq n \leq 10)$ of $t_{6}\left(P_{6}, q_{P_{6}} ; z\right)$ :

$$
\begin{array}{r}
1 \\
0 \\
18480 \\
0 \\
12803590800 \\
0 \\
-817993722627081000 \\
0 \\
-156078929845326558019950000 \\
0 \\
122859953407720110679241179380345000
\end{array}
$$

and their factorizations:

1

$$
2^{4} \cdot 3 \cdot 5 \cdot 7 \cdot 11
$$$$
0
$$$$
2^{4} \cdot 3^{2} \cdot 5^{2} \cdot 7 \cdot 11^{2} \cdot 13 \cdot 17 \cdot 19
$$$$
0
$$$$
-2^{3} \cdot 3^{3} \cdot 5^{3} \cdot 7^{3} \cdot 11 \cdot 13 \cdot 17 \cdot 19 \cdot 23 \cdot 29 \cdot 47 \cdot 61
$$

0 $-2^{4} \cdot 3^{4} \cdot 5^{5} \cdot 7^{3} \cdot 11^{2} \cdot 13 \cdot 17 \cdot 19^{2} \cdot 23 \cdot 29 \cdot 31 \cdot 41 \cdot 13729$ 0 $2^{3} \cdot 3^{5} \cdot 5^{4} \cdot 7^{4} \cdot 11^{4} \cdot 13^{2} \cdot 17 \cdot 19 \cdot 23 \cdot 29 \cdot 31 \cdot 37 \cdot 41 \cdot 43 \cdot 661 \cdot 59107$ 0

6. Transcendence Classes of adapted local CONStants at Elliptic POINTS AND AT SCM-POINTS

Definition 6.1. Let $K$ be any subfield of $\mathbb{C}$. Given $a, b \in \mathbb{C}^{*}$, we write $a \sim_{K} b$ for the equivalence relation defined by $a b^{-1} \in \bar{K}^{*}$. We say in this case that $a$ and $b$ have the same transcendence class over $K$ and we identify $\mathbb{C}^{*} / \bar{K}^{*}$ with the group of all transcendence classes over $K$ of non-zero complex numbers. When $K=\mathbb{Q}$, we simply write $a \sim b$ and say that $a, b$ have the same transcendence class. Note that the transcendence class of 1 is $\overline{\mathbb{Q}}^{*}$, and that $0, \infty$ are excluded from our considerations. Hence our notation differs from that of [23]. 
The following result is useful to compare the transcendence classes of local constants adapted to automorphic functions for different groups.

Proposition 6.2. Let $\Gamma, \Gamma^{\prime} \subseteq \mathbf{G L}^{+}(2, \mathbb{R})$ be commensurable Fuchsian groups. Let $t, t^{\prime}$, be non-constant automorphic functions for $\Gamma, \Gamma^{\prime}$. Then the functions $t, t^{\prime}$ are algebraically dependent over a field $K \subseteq \mathbb{C}$. Suppose, moreover, that $t(P), t^{\prime}(P) \in K \cup\{\infty\}$ for a point $P \in \mathcal{H}$. Then

$$
k(P, \Gamma, t) \sim_{K} k\left(P, \Gamma^{\prime}, t^{\prime}\right) .
$$

Proof. We prove only the second claim, since the first one is well known. By using proposition 4.2, we can certainly assume that $t(P)=t^{\prime}(P)=0$. Let $F\left(t, t^{\prime}\right)=0$ for $F(X, Y) \in K[X, Y]-K$. We endow the variable $X$ with the weight $r e_{P}$ and the variable $Y$ with the weight $r^{\prime} e_{P}^{\prime}$, where $e_{P}=\# \bar{\Gamma}_{P}, e_{P}^{\prime}=\# \bar{\Gamma}_{P}^{\prime}$. The polynomial $F$ can be written as

$$
F(X, Y)=\sum_{s=0}^{N} F_{s}(X, Y), \quad F_{s}(X, Y)=\sum_{m r e_{P}+n r^{\prime} e_{P}^{\prime}=s} a_{m, n} X^{m} Y^{n}, \quad a_{m, n} \in K,
$$

so that $F_{s}$ stands for an isobaric polynomial of weight $s$. Let $s_{0}>0$ be the least weight for which $F_{s_{0}}(X, Y) \neq 0$. Let $q_{P}, q_{P}^{\prime}$ be local parameters at $P, P^{\prime}$ adapted to the functions $t, t^{\prime}$ :

$$
q_{P}(z)=\left(k_{P} \frac{z-P}{z-\bar{P}}\right)^{e_{P}}, \quad q_{P}^{\prime}(z)=\left(k_{P}^{\prime} \frac{z-P}{z-\bar{P}}\right)^{e_{P}^{\prime}} .
$$

We have

$$
0=F\left(t\left(q_{P}\right), t^{\prime}\left(q_{P}^{\prime}\right)\right)=F_{s_{0}}\left(t\left(q_{P}\right), t^{\prime}\left(q_{P}^{\prime}\right)\right)+O\left(\left(\frac{z-P}{z-\bar{P}}\right)^{s_{0}+1}\right) .
$$

From this we deduce that

$$
\sum_{m r e_{P}+n r^{\prime} e_{P}^{\prime}=s_{0}} a_{m, n} k_{P}^{r m e_{P}} k_{P}^{\prime r^{\prime} n e_{P}^{\prime}}=0, \quad a_{m, n} \in K
$$

This gives a non-trivial relation of the form

$$
\sum_{j=0}^{s_{0}} a_{j}\left(\frac{k_{P}}{k_{P}^{\prime}}\right)^{j}=0, \quad a_{j} \in K,
$$

which clearly forces $k_{P} \sim_{K} k_{P}^{\prime}$.

In what follows, we change the notation slightly and $k(P, X, t)$ will also stand for $k(P, \Gamma, t)$ if $X=X(\Gamma)$ is the canonical model of the complex projective nonsingular curve defined by $\Gamma$. 
Next we obtain representatives of the transcendence classes of the local constants adapted to the function $t_{6}$ at the elliptic points and at the SCM-points for $\Gamma_{6}$.

Theorem 6.3. The following relations hold:

$$
\begin{aligned}
& k\left(P_{1}, X_{6}, t_{6}\right) \sim k\left(P_{3}, X_{6}, t_{6}\right) \sim k\left(P_{5}, X_{6}, t_{6}\right) \sim k\left(P_{6}, X_{6}, t_{6}\right) \sim \frac{\Gamma(1 / 4) \Gamma(5 / 12)}{\Gamma(3 / 4) \Gamma(11 / 12)}, \\
& k\left(P_{2}, X_{6}, t_{6}\right) \sim k\left(P_{4}, X_{6}, t_{6}\right) \sim \frac{\Gamma(1 / 3)^{2} \Gamma(7 / 12)}{\Gamma(2 / 3)^{2} \Gamma(11 / 12)}, \\
& k\left(P_{0}, X_{6}, t_{6}\right) \sim k\left(P_{7}, X_{6}, t_{6}\right) \sim k\left(P_{8}, X_{6}, t_{6}\right) \sim \frac{\Gamma(7 / 24) \Gamma(11 / 24)}{\Gamma(19 / 24) \Gamma(23 / 24)} .
\end{aligned}
$$

Proof. Consider the 4 -fold covering $\Gamma_{6} \backslash \mathcal{H} \rightarrow \Gamma_{6}^{+} \backslash \mathcal{H}$. The two classes in the first quotient defined by the points $P_{1}, P_{3}, P_{5}, P_{6}$ are identified with the class in the second quotient defined by the point $P_{6}$. Similarly, the two classes defined by $P_{2}, P_{4}$ are identified with the class defined by $P_{4}$, and the two classes defined by $P_{0}, P_{7}, P_{8}$ are identified with the class defined by $P_{0}$. Thus the result follows from the equations in propositions $2.1,4.2$ and 6.2 , and table 7 .

Let $H\left(d f^{2}\right)$ be a complete set of non-equivalent positive definite primitive binary quadratic forms $a_{j} X^{2}+b_{j} X Y+c_{j} Y^{2}$ with discriminant $b_{j}^{2}-4 a_{j} b_{j}=d f^{2}$, where $d$ is a fundamental discriminant and $f \geq 1$ is an integer, and let $h=h\left(d f^{2}\right)$ be the class number. The zeros in $\mathcal{H}$ of these forms,

$$
\tau_{j}=\frac{-b_{j}+f \sqrt{d}}{2 a_{j}}, \quad 1 \leq j \leq h,
$$

define complex multiplication points by $\mathbb{Q}(\sqrt{d})$ on the modular curve $X_{0}(1)$.

We consider the discriminant modular form $\Delta(z):=e^{2 \pi i z} \prod_{n=1}^{\infty}\left(1-e^{2 \pi i n z}\right)^{24}$, where $z \in \mathcal{H}$.

Next we recall the formula of Chowla-Selberg (cf. [17]). For the case $f=1$, it produces an expression for the product of the $h(d)$ values $\Delta\left(\tau_{j}\right)$ in terms of values of Euler's $\Gamma$-function at rational arguments:

$$
\prod_{j=1}^{h} \Delta\left(\tau_{j}\right)=\frac{1}{(2 \pi|d|)^{6 h}} \prod_{j=1}^{h} a_{j}^{6} \prod_{m=1}^{|d|}\left\{\Gamma\left(\frac{m}{|d|}\right)^{\left(\frac{d}{m}\right)}\right\}^{3 w} .
$$

Here $\left(\frac{d}{m}\right)$ is the Kronecker symbol, and $w= \begin{cases}6, & \text { if } d=-3, \\ 4, & \text { if } d=-4, \\ 2, & \text { otherwise. }\end{cases}$ 
Definition 6.4. For a given fundamental discriminant $d<0$, we put

$$
\pi_{d}:=\prod_{m=1}^{|d|}\left\{\Gamma\left(\frac{m}{|d|}\right)^{\left(\frac{d}{m}\right)}\right\}^{\frac{w}{2 h}} .
$$

Proposition 6.5. We have

$$
k\left(P_{0}, X_{6}, t_{6}\right) \sim \pi_{-24}, \quad k\left(P_{3}, X_{6}, t_{6}\right) \sim \pi_{-4}, \quad k\left(P_{4}, X_{6}, t_{6}\right) \sim \pi_{-3} .
$$

Proof. It suffices to observe that

$$
\begin{gathered}
\left(\frac{\pi_{-24}}{k\left(P_{0}, X_{6}, t_{6}\right)}\right)^{2}=-4,\left(\frac{\pi_{-4}}{k\left(P_{3}, X_{6}, t_{6}\right)}\right)^{4}=\frac{-256}{3} \\
\left(\frac{\pi_{-3}}{k\left(P_{4}, X_{6}, t_{6}\right)}\right)^{12}=\frac{-531441}{4}
\end{gathered}
$$

Theorem 6.6. The number $\pi_{d}$ is transcendental.

Proof. To begin with we recall the expression of the modular function $j(z)$ as a rational function of the modular function $k(z)$ :

$$
j(z)=2^{8} \frac{\left(k(z)^{4}-k(z)^{2}+1\right)^{3}}{k(z)^{4}\left(k(z)^{2}-1\right)^{2}} .
$$

Let $\tau \in \mathbb{Q}(\sqrt{d}) \cap \mathcal{H}$. By the classical complex multiplication theory, $j(\tau) \in \overline{\mathbb{Q}}$ and, thus, $k(\tau) \in \overline{\mathbb{Q}}$. The elliptic curve with Jacobi model

$$
E: Y^{2}=\left(1-X^{2}\right)\left(1-k(\tau) X^{2}\right)
$$

is defined over $\overline{\mathbb{Q}}$, has complex multiplication by $\mathbb{Q}(\sqrt{d})$. Let $\langle 4 K(\tau), 4 K(\tau) \tau\rangle$ be is its period lattice. The Weierstrass model of $E$ is also defined over $\overline{\mathbb{Q}}$. Now, by a theorem due to Chudnovsky (cf. [8] and [25]), for every non-zero period $\lambda \in \Lambda$, the numbers $\lambda$ and $\pi$ are algebraically independent. Since

$$
\Delta(\tau) \sim\left(\frac{K(\tau)}{\pi}\right)^{12}, \quad \Delta\left(\tau_{i}\right) \sim \Delta\left(\tau_{j}\right), \quad \text { for } 1 \leq j \leq h,
$$

and after Chowla-Selberg we know that $K(\tau) \sim\left(\pi \cdot \pi_{d}\right)^{1 / 2}$, it follows that $\pi_{d}$ is transcendental.

Theorem 6.7. The local constants adapted to the functions $t_{6}, t_{6}^{(2)}, t_{6}^{(3)}, t_{6}^{(6)}, t_{6}^{+}$at the elliptic points and at the SCM-points are transcendental. They are displayed in tables 6 and $\%$.

Once we have understood the nature of the local constants at the elliptic points and at the SCM-points of the curve $X_{6}$, we may ask whether the results in theorem 6.7 remain true for every CM-point. We will see that this is the case in section 7 . 


\section{Arithmetical properties of the Canonical models}

As we recalled in the proof of theorem 6.6 , given an imaginary quadratic field $K$ of discriminant $d$, there exists an elliptic curve $E$ defined over $\overline{\mathbb{Q}}$ with complex multiplication by $K$ and a $\overline{\mathbb{Q}}$-rational holomorphic differential form $\omega$ in $E$ such that

$$
\int_{c} \omega \sim\left(\pi \cdot \pi_{d}\right)^{1 / 2}
$$

for all 1-cycles $c$ on $E$. Moreover, we can choose an isomorphism $\varphi: K \simeq$ $\operatorname{End}(E) \otimes \mathbb{Q}$ so that $\varphi(a)^{*} \omega=a \omega$, for every $a \in K$.

Assume now that the field $K$ is embeddable in $\mathrm{H}_{6}$ and let $P \in \mathcal{H}$ be a CM-point by $K$. We can choose an embedding $\varphi: K \hookrightarrow \mathrm{H}_{6}$ by imposing that

$$
\Phi(\varphi(a))\left[\begin{array}{l}
P \\
1
\end{array}\right]=a\left[\begin{array}{l}
P \\
1
\end{array}\right], \quad \text { for every } a \in K .
$$

Theorem 7.1. Let $\Gamma$ be a Fuchsian group commensurable with $\Gamma_{6}$. Let $t$ be a nonconstant $\overline{\mathbb{Q}}$-rational function of the canonical model $X(\Gamma)$ of the curve associated to $\Gamma$. Suppose that $P \in \mathcal{H}$ is a CM-point by a quadratic field $K$ of discriminant d. Then

$$
k(P, X(\Gamma), t) \sim \pi_{d} .
$$

Proof. We begin by considering the case $t=t_{6}^{+}$. Taking into account definition 4.1 and propositions $6.2,6.5$, the following relations hold at the elliptic points for $\Gamma_{6}^{+}$:

$$
k\left(P_{0}, X_{6}^{+}, t_{6}^{+}\right) \sim \pi_{-24}, \quad k\left(P_{4}, X_{6}^{+}, t_{6}^{+}\right) \sim \pi_{-3}, \quad k\left(P_{6}, X_{6}^{+}, t_{6}^{+}\right) \sim \pi_{-4} .
$$

If $P$ is a CM-point which is not elliptic for $\Gamma_{6}^{+}$, then $\operatorname{div}\left(t_{6}^{+}-t_{6}^{+}(P)\right)=(P)-\left(P_{6}\right)$ since, by construction of the function $t_{6}^{+}, \operatorname{div}\left(t_{6}^{+}\right)=\frac{2}{2}\left(P_{0}\right)-\frac{4}{4}\left(P_{6}\right)$. Therefore, $D\left(t_{6}^{+}, P\right) \neq 0$ and by definition 4.1

$$
k\left(P, X_{6}^{+}, t_{6}^{+}\right)=2 i \operatorname{Im}(P) D\left(t_{6}^{+}, P\right) \sim D\left(t_{6}^{+}, P\right) .
$$

Since $t_{6}^{+}$is a $\overline{\mathbb{Q}}$-rational function for $X_{6}$ and $D\left(t_{6}^{+}, P\right) \neq 0$, we may now apply Shimura [23], theorem 7.1 to deduce that $D\left(t_{6}^{+}, P\right) \sim \pi_{d}$.

The claim for any function $t$ as in the statement of the theorem follows from proposition 6.2. Therefore,

$$
k(P, X(\Gamma), t) \sim k\left(P, X_{6}^{+}, t_{6}^{+}\right) \sim \pi_{d} .
$$

Definition 7.2. Let $K$ be an imaginary quadratic field of discriminant $d$. Suppose that $K$ is embeddable in $\mathrm{H}_{6}$ and let $P \in \mathcal{H}$ be a CM-point by $K$. Let $\Gamma \subseteq \mathbf{G L}^{+}(2, \mathbb{R})$ be a Fuchsian group commensurable with $\Gamma_{6}$. 
(i) An arithmetical local parameter at $P$ under the $\Gamma$-action is any function $q_{P}(z):=\left(k \frac{z-P}{z-\bar{P}}\right)^{e_{P}}, \quad e_{P}=\# \bar{\Gamma}_{P}, \quad$ for which $k \sim \pi_{d}$.

(ii) A $\Gamma$-automorphic function is called arithmetical at a CM-point $P$ if its series expansion around $P$ in powers of an arithmetical local parameter $q_{P}(z)$ has algebraic coefficients.

(iii) A $\Gamma$-automorphic function is called arithmetical if it is arithmetical at every CM-point.

Putting all this together, we obtain the following theorem which tells us that, at least for $D=6$, the $\overline{\mathbb{Q}}$-rational automorphic functions of the canonical models $X(\Gamma)$ are precisely the arithmetical ones.

Theorem 7.3. Let $\Gamma$ be a Fuchsian group of finite index in $\Gamma_{6}^{+}$. For a $\Gamma$ automorphic function $t$, the following conditions are equivalent:

(i) $t \in \overline{\mathbb{Q}}(X(\Gamma))$.

(ii) $t$ is arithmetical at a $C M$-point $P$.

(iii) $t$ is arithmetical.

Proof. Since $t_{6}^{+}$defines the canonical model for $X_{6}^{+}$, we have that $t_{6}^{+}(P) \in$ $\overline{\mathbb{Q}} \cup\{\infty\}$, for each CM-point $P$. The fact that the coefficients of the differential equation satisfied by $t_{6}^{+}$are algebraic (see table 5 ) together with theorem 7.1 show that $t_{6}^{+}$will be arithmetical at every CM-point $P$.

Given our $\Gamma$-automorphic function $t$, we consider the polynomial $F\left(T_{1}, T_{2}\right)$ in $\mathbb{C}\left(T_{1}\right)\left[T_{2}\right]$ such that $F\left(t_{6}^{+}, T_{2}\right)$ is the monic irreducible polynomial of $t$ over $\mathbb{C}\left(t_{6}^{+}\right)$.

Assume now that $t$ satisfies (i). Then $t(P) \in \overline{\mathbb{Q}} \cup\{\infty\}$ for any CM-point $P$. Since we already know that $t_{6}^{+}$is arithmetical at every CM-point $P$ and these points are dense in $\mathcal{H}$, we must have that $F \in \overline{\mathbb{Q}}\left(T_{1}\right)\left[T_{2}\right]$. It turns out that the function $t$ is arithmetical at every point CM-point $P$ so that (iii) is fulfilled.

Suppose now that (ii) is satisfied, with $t$ being arithmetical at $P, t(P) \neq \infty$; in particular $t(P) \in \overline{\mathbb{Q}}$. From $F\left(t_{6}^{+}(P), t(P)\right)=0$, we deduce that $F \in \overline{\mathbb{Q}}\left(T_{1}\right)\left[T_{2}\right]$. This implies that $t(P) \in \overline{\mathbb{Q}}$ and, thus, $t$ will satisfy (i). If $t(P) \neq \infty$, we just change $t$ by $1 /(1-t)$ and repeat the argument for this function.

It is worth pointing out that the assertions in theorem 7.3 also hold in the modular case (corresponding to $D=1$ ): the $\overline{\mathbb{Q}}$-rational functions of the modular curves are visible through their series expansions at infinity (in terms of the parameter $q_{\infty}(z)=e^{2 \pi i z}$, or through their series expansions around any other 
complex multiplication point (once the right arithmetical local parameters have been chosen, cf. [5]).

We may conclude from our examples that the lack of cusps does not preclude the existence of an arithmetical theory of automorphic functions.

\section{REFERENCES}

[1] Alsina, M.; Bayer, P.: Quaternion orders, quadratic forms and Shimura curves. CRM Monograph Series 22. AMS, 2004. xvi + 196 pp.

[2] Baba, S.; Granath, H.: Genus 2 curves with quaternionic multiplication. Max-PlanckInstitut für Mathematik. Preprint Series 2005 (18).

[3] Bayer, P.: Uniformization of certain Shimura curves. In Differential Galois Theory, T. Crespo and Z. Hajto (eds.). Banach Center Publications 58 (2002), 13-26.

[4] Bayer, P.; Guàrdia, J.: On equations defining fake elliptic curves. J. Théor. Nombres Bordeaux 17 (2005), no. 1, 57-67.

[5] Bayer, P.; Travesa, A.: Uniformization of triangle modular curves. Publ. Mat., extra vol. (2007), 43-106.

[6] Bayer, P.; Travesa, A.: Uniformizing functions for certain Shimura curves, in the case $D=6$, Acta Arith. 126, no. 4 (2007), 315-339.

[7] Bolza, O.: Darstellung der rationalen ganzen Invarianten der Binärform sechsten Grades durch die Nullwerthe der zugehörigen $\vartheta$-Functionen. Math. Ann. 30 (1887), 478-495.

[8] Chudnovsky, G. V.: Contributions to the theory of transcendental numbers. Mathematical Surveys and Monographs, 19. American Mathematical Society, Providence, RI, 1984. $\mathrm{xi}+450 \mathrm{pp}$.

[9] Cohen, H.; Frey, G.: Handbook of elliptic and hyperelliptic curve cryptography, Discrete Math. Appl. (Boca Raton), Chapman \& Hall/CRC, Boca Raton, FL, 2006. xxxiv + 808 pp.

[10] Hashimoto, K.: Explicit form of quaternion modular embeddings. Osaka J. Math. 32 (1995), no. 3, 533-546.

[11] Hashimoto, K.: Murabayashi, N.: Shimura curves as intersections of Humbert surfaces and defining equations of QM-curves of genus two. Tohoku Math. J.(2) 47 (1995), no. 2, 271-296.

[12] Igusa, J.: Arithmetic variety of moduli for genus two. Ann. of Math. (2) 72 (1960), 612-649.

[13] Ribet, K. A.: On modular representations of $\operatorname{Gal}(\overline{\mathbb{Q}} / \mathbb{Q})$ arising from modular forms. Invent. Math. 100 (1990), no. 2, 431-476.

[14] Rodriguez-Villegas, F.: Explicit models of genus 2 curves with split CM. In Algorithmic number theory (Leiden, 2000), W. Bosma (ed.). Lecture Notes in Comput. Sci. 1838. Springer, 2000, 505-513.

[15] Rotger, V.: Modular Shimura varieties and forgetful maps. Trans. Amer. Math.Soc. 356 (2004), no. 4, 1535-1550.

[16] Rotger, V.: Shimura curves embedded in Igusa's threefold. In Modular curves and abelian varieties, J. Cremona, J-C. Lario, J. Quer, K. Ribet (eds.). Progr. Math. 224, Birkhäuser, Basel, 2004, 263-276. 
[17] Selberg, A.; Chowla, S.: On Epstein's zeta-function. J. Reine Angew. Math. 227 (1967), 86-110.

[18] Serre, J-P.: A course in arithmetic. Translated from the French. Graduate Texts in Mathematics, No. 7. Springer, 1973. viii+115 pp.

[19] Serre, J-P.: Sur les représentations modulaires de degré 2 de $\operatorname{Gal}(\overline{\mathbf{Q}} / \mathbf{Q})$. Duke Math. J.54 (1987), no. 1, 179-230. CEuvres. Collected papers, Vol. III, 1972-1984, 133. Springer, 1986. iv $+728 \mathrm{pp}$.

[20] Serre, J-P.: Travaux de Wiles (et Taylor, ...). I. Séminaire Bourbaki, Vol. 1994/95. Astérisque No. 237 (1996), Exp. No. 803, 5, 319-332. CEuvres. Collected papers, Vol. IV, 1985-1998, 168. Springer, 2000. viii+657 pp.

[21] Shimura, G.: Construction of class fields and zeta functions of algebraic curves. Annals of Math. 85 (1967), 58-159. Collected papers, Vol. II, 1967-1977, 67b. Springer, 2002. xiv $+831 \mathrm{pp}$.

[22] Shimura, G.: Introduction to the Arithmetic Theory of Automorphic Functions. Iwanami Shoten and Princeton University Press, 1971. xiv +267 pp.

[23] Shimura, G.: Automorphic forms and periods of abelian varieties J. Math. Soc. Japan 31 (1979), 561-592. Collected papers, Vol. III, 1978-1988, 79a. Springer, 2003. xiv+924 pp.

[24] Shimura, G.: Abelian varieties with complex multiplication and modular functions. Princeton Mathematical Series, 46. Princeton University Press, Princeton, NJ, 1998. xvi+218 pp.

[25] Waldschmidt, M.: Nombres transcendants et groupes algébriques. With appendices by Daniel Bertrand and Jean-Pierre Serre. Astérisque, 69-70. Société Mathématique de France, Paris, 1979. 218 pp.

\section{P. Bayer}

Facultat de Matemàtiques. Universitat de Barcelona. Gran Via de les Corts Catalanes 585. E-08007, Barcelona

E-mail: bayer@ub.edu

\section{A. Travesa}

Facultat de Matemàtiques. Universitat de Barcelona. Gran Via de les Corts Catalanes 585. E-08007, Barcelona

E-mail: travesa@ub.edu 